\title{
Implementación de ciclo de mejora docente en la asignatura Fisioterapia en Atención Temprana del Grado en Fisioterapia
}

Elena Piñero PINTO

Universidad de Sevilla

Departamento de Fisioterapia

epinero@us.es

D.O.I.: http://dx.doi.org/10.12795/JDU.2018.i01.32

Pp.: 566-581

\section{Resumen}

El Ciclo de Mejora Docente (CMD) ha sido llevado a cabo en la asignatura de Fisioterapia en Atención Temprana (AT), asignatura optativa del Grado en Fisioterapia. Se ha realizado en un total de 7 horas, programadas en dos semanas lectivas. Basándonos en el mapa de contenidos, problemas y actividades, hemos realizado un cuestionario inicial para conocer los esquemas mentales de los alumnos y adaptarnos a ellos. Se han llevado a cabo 5 actividades de aprendizaje diferentes para abordar todos los contenidos conceptuales, procedimentales y actitudinales. Posteriormente se ha administrado un cuestionario final para observar el aprendizaje del alumnado, y una encuesta de evaluación del ciclo de mejora y de la docente para analizar la visión y perspectiva del alumnado. En general, la progresión de los alumnos y 
su grado de implicación y participación han sido bastante altos, por lo que ha resultado una experiencia enriquecedora para todos.

Palabras Clave: Fisioterapia en Atención Temprana, Grado en Fisioterapia, Docencia Universitaria, Experimentación Docente Universitaria.

\section{Breve descripción del contexto}

La asignatura "Fisioterapia en Atención Temprana" es optativa de segundo curso del Grado de Fisioterapia. Durante el primer cuatrimestre, los alumnos del Grado en Fisioterapia cursan otra asignatura denominada "Medicina del Desarrollo", la cual antecede en contenidos a la asignatura donde aplicamos el Ciclo de Mejora Docente (CMD), ya que se estudian las patologías más frecuentes de la primera infancia desde una visión global y holística.

Nuestra asignatura consiste en aprender a evaluar e intervenir en los distintos trastornos del desarrollo de la primera infancia, desde las cuatro áreas básicas del desarrollo (cognitiva, motora, lenguaje y social) con una perspectiva desde la Fisioterapia. Actualmente, los equipos básicos de Atención Temprana (AT) están formados por profesionales sanitarios de la psicología, logopedia y fisioterapia, por lo que se hace imprescindible esta asignatura en el Grado en Fisioterapia.

\section{Diseño del Ciclo de Mejora Docente}

\section{Mapa de contenidos y problemas clave}

Los contenidos abordados los vamos a dividir, para su mejor comprensión y análisis, en:

- Conceptuales:

- Diagnóstico de Fisioterapia en Atención Temprana. 
- Características del desarrollo en los trastornos y situaciones de riesgo en la primera infancia.

- Técnicas de intervención específicas en Fisioterapia Pediátrica.

- Señales de alarma en los primeros años de vida en los distintos trastornos del desarrollo.

- Actitudinales:

- Cooperación y coordinación con familia y otros profesionales.

- Trabajo en equipo interdisciplinar.

- Toma de decisiones.

- Límite de las competencias profesionales, honestidad y humildad.

- Ética y responsabilidad.

- Procedimentales:

- Implementación de la evaluación en AT.

- Manejo del diagnóstico de Fisioterapia.

- Manipulación del niño: cambios posturales, transferencias, facilitación de los hitos del desarrollo.

- Planificación y organización profesional.

Para organizar estos contenidos en niveles de profundidad y relacionarlos con las actividades que hemos desarrollado, realizamos un mapa que aúna contenidos, actividades y problemas a trabajar con el alumnado (Figura 1).

En el mapa de contenidos, problemas y actividades podemos ver una progresión desde la parte superior a la inferior y de izquierda a derecha del mapa. En los cuadros rectangulares de colores observamos los contenidos conceptuales, de los que se desprenden los principales problemas a resolver, que se encuentran en los globos de color amarillo. De éstos, se desprenden, a su vez, los contenidos procedimentales y actitudinales marcados en listado al lado o debajo. Finalmente, con el símbolo del lápiz se marcan todas las actividades previstas en el ciclo de mejora. 


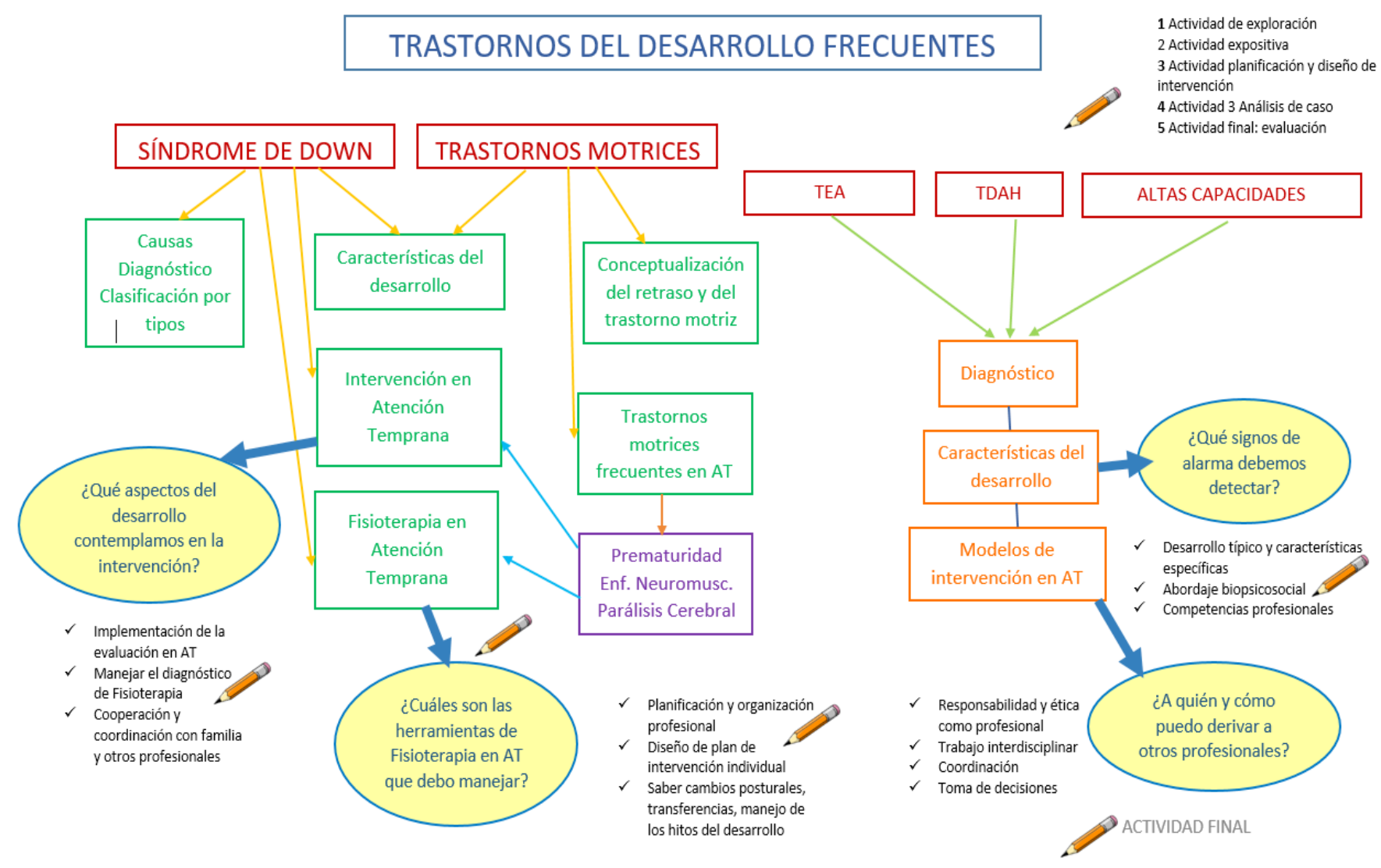

Figura 1. Mapa de contenidos, problemas y actividades.

Jornadas de Formación e Innovación Docente del Profesorado | № 1 (2018)

(c) (i) E E Esta obra se distribuye con la licencia Creative Commons 


\section{Modelo metodológico posible y secuencia de actividades}

El esquema diseñado, en función del modelo metodológico que hemos empleado (De Alba y Porlán, 2017), es el recogido en la Figura 2.

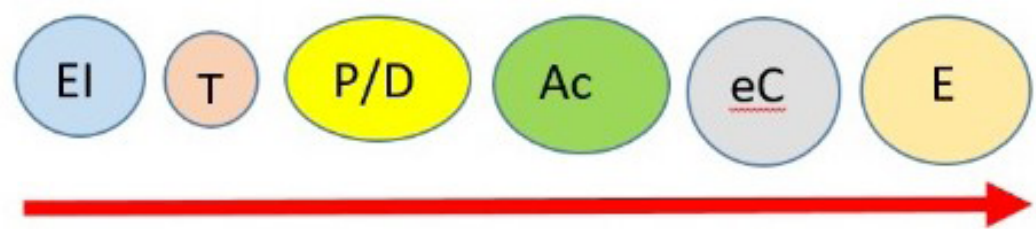

Figura 2. Modelo metodológico posible.

Este modelo de aprendizaje posible contempla la secuencia siguiente: Exploración de ideas de los alumnos (EI) à Teoría (en debate) (T) à Planificación y diseño de programación (P/D) à Análisis de Caso y establecimiento de señales de alarma (Ac) à Elaboración de Conclusiones (eC) à Evaluación (E).

La justificación de este modelo parte del conocimiento del nivel inicial de los alumnos para, a partir de ahí, debatir en grupo los conceptos teóricos de las características del desarrollo de los distintos trastornos. Una vez conocidos, así como las herramientas de intervención en el desarrollo motriz (contenido visto en temas anteriores), los alumnos han elegido un trastorno del desarrollo, con una edad específica, y realizan una planificación de objetivos de intervención, así como de las técnicas y herramientas usadas para llevarlo a cabo. Posteriormente se ha expuesto en clase cada uno de ellos y se ha completado con información de la profesora y otros alumnos.

La siguiente actividad consistió en analizar un caso real de un niño con trastorno de espectro autista y determinar los signos de alarma en su desarrollo, para debatir en grupo qué posible trastorno podría tener y qué podemos hacer desde la fisioterapia. Después realizamos unas preguntas finales para elaborar las conclusiones: ¿En 
qué trastornos del desarrollo tiene la fisioterapia un papel clave? ¿Cuáles son las funciones del fisioterapeuta en Atención Temprana en dichos trastornos? Se debaten en grupo de viva voz. Por último, se realiza la evaluación.

\section{Secuencia de actividades}

La secuencia de actividades llevada a cabo en este CMD se recoge en la Tabla 1, a continuación. 
Tabla 1. Secuencia de actividades del Ciclo de Mejora.

\begin{tabular}{|c|c|c|}
\hline Descripción de la actividad & $\begin{array}{l}\text { Sentido educativo } \\
\text { de la actividad }\end{array}$ & $\begin{array}{l}\text { Contenidos desarro- } \\
\text { llados en la actividad }\end{array}$ \\
\hline $\begin{array}{l}\text { Actividad 1. Exploración de ideas. } \\
\text { Se realiza un cuestionario de explora- } \\
\text { ción de ideas iniciales de los alumnos } \\
\text { acerca de sus creencias, prejuicios y } \\
\text { conocimientos sobre el abordaje desde } \\
\text { la Atención Temprana de los trastornos } \\
\text { más frecuentes del desarrollo. } \\
\text { Tiempo estimado: } 30 \text { minutos. }\end{array}$ & $\begin{array}{l}\text { El sentido de esta } \\
\text { actividad es co- } \\
\text { nocer los conoci- } \\
\text { mientos e ideas } \\
\text { iniciales, así como } \\
\text { poder adaptar el } \\
\text { resto del conte- } \\
\text { nido al nivel real } \\
\text { del alumnado. } \\
\end{array}$ & $\begin{array}{l}\text { - Evaluación inicial. } \\
\text { - Ideas iniciales. }\end{array}$ \\
\hline $\begin{array}{l}\text { Actividad 2. Teoría / Debate. } \\
\text { A partir de situar a los alumnos en los } \\
\text { trastornos del desarrollo o riesgos de } \\
\text { padecerlos, se pretende asociar estos } \\
\text { conceptos a su actitud y conocimiento } \\
\text { frente a qué pueden aportar ellos, } \\
\text { como profesionales, a estos niños y } \\
\text { qué harian por ellos desde la Fisiote- } \\
\text { rapia en AT. Se plantean dos preguntas } \\
\text { que deben reflexionar de forma grupal } \\
\text { tras la visualización de un video pro- } \\
\text { pio de la profesora realizando una se- } \\
\text { sión de AT con un bebé con síndrome } \\
\text { de Down: } \\
\text { - ¿Qué características del desarrollo } \\
\text { tiene el bebé del vídeo y que son } \\
\text { frecuentes en su trastorno del } \\
\text { desarrollo? } \\
\text { - ¿Qué sintomatología del niño con } \\
\text { Trastorno del Espectro Autista (TEA) te } \\
\text { llama la atención del vídeo? } \\
\text { Se trata de comentar, en grupo, cuáles } \\
\text { son las características del desarrollo } \\
\text { de los trastornos que vamos a estudiar } \\
\text { para intervenir desde la AT. } \\
\text { Tiempo estimado: } 1 \text { hora y } 30 \text { minutos. }\end{array}$ & $\begin{array}{l}\text { Se trata de facili- } \\
\text { tarles a los alum- } \\
\text { nos los principales } \\
\text { síntomas de los } \\
\text { trastornos del de- } \\
\text { sarrollo y situacio- } \\
\text { nes de riesgo de } \\
\text { padecerlos, con- } \\
\text { tenidos que va- } \\
\text { mos a trabajar en } \\
\text { el resto de activi- } \\
\text { dades, para desa- } \\
\text { rrollar una actitud } \\
\text { de búsqueda y re- } \\
\text { flexiva sobre el } \\
\text { papel de la fisio- } \\
\text { terapia en estos } \\
\text { trastornos. Se trata } \\
\text { de anticipar con- } \\
\text { ceptos teóricos, } \\
\text { haciéndolo desde } \\
\text { el propio razona- } \\
\text { miento y reflexión. }\end{array}$ & $\begin{array}{l}\text { - Características del } \\
\text { desarrollo. } \\
\text { - Trastornos frecuen- } \\
\text { tes en la primera } \\
\text { infancia. } \\
\text { - Signos y síntomas } \\
\text { de alarma en los dis- } \\
\text { tintos trastornos. } \\
\text { - Planificación en el } \\
\text { diseño de un pro- } \\
\text { grama de Atención } \\
\text { Temprana. } \\
\text { - Unificación de con- } \\
\text { ceptos y conoci- } \\
\text { mientos de temas } \\
\text { anteriores (evalua- } \\
\text { ción y desarrollo e in- } \\
\text { tervención por áreas) } \\
\text { a los propios de este } \\
\text { tema. }\end{array}$ \\
\hline
\end{tabular}


Actividad 3. Actividad de planificación y diseño de intervención.

Se trata de realizar un diseño de plan de intervención individual en función de las características del desarrollo de los trastornos que hemos estudiado en la actividad anterior. Se les da a elegir un trastorno de los que vamos a desarrollar, y cada alumno tendrá que trabajar sobre el diseño de la intervención de un caso concreto. Esta tarea se inicia en clase y al final de la misma debe estar terminada. Se les facilitará una serie de cuestiones:

- ¿Qué características del desarrollo tiene este trastorno?

- ¿Sobre qué características puede trabajar el fisioterapeuta en AT?

- Si podemos trabajar aspectos del desarrollo, ¿cuáles serían los objetivos de intervención en este caso?

- ¿Qué técnicas o herramientas de intervención crees que serían los más adecuados?

Tiempo estimado: 3 horas. Actividad 4. Análisis de caso.

Esta actividad consiste en visualizar un vídeo de un niño en una tarea dirigida [https://www.youtube.com/watch?$\left.v=r 0-\_c-P j 3 H E\right]$. Los alumnos desconocen el trastorno que tiene (TEA) y tendrán que describir, por escrito, qué signos de alarma observan respecto al desarrollo general. Tras hablar de los signos y características de estos trastornos en grupo, se debate qué puede hacer el fisioterapeuta en dicho trastorno.

Tiempo estimado: 1 hora.
Es la tarea más compleja de todo el tema. Tendrán que unificar aprendizajes de temas anteriores (evaluación, desarrollo e intervención por áreas y planificación en Atención Temprana) con los aprendizajes del tema actual (trastornos del desarrollo). En esta actividad el alumno aprenderá a organizarse y planificar una intervención individualizada, de manera que se adapte a cada niño y trastorno.

Se pretende trabajar el desarrollo de habilidades críticas en AT, la detección temprana y la intervención temprana. También se trata de que el alumno sea capaz de saber sus competencias y sus limitaciones dentro de la AT.
- Capacidad de organización y relación de aprendizajes anteriores con el tema actual.

- Conceptos propios de los trastornos del desarrollo estudiados.

- Integra-

ción en equipo interdisciplinar. - Creatividad y planificación en el diseño de tareas y actividades.

- Resolución de casos reales aplicados a la Atención Temprana.

- Signos de alarma en los distintos trastornos.

- Capacidad crítica de observación.

- Derivación y ámbito de aplicación de la Fisioterapia en Atención Temprana. 
Actividad 5. Elaboración de conclusiones.

Se lanzan dos preguntas al grupo clase completo para debatir las respuestas: ¿En qué trastornos del desarrollo tiene la fisioterapia un papel clave? ¿Cuáles son las funciones del fisioterapeuta en AT en dichos trastornos?

Tiempo estimado: 30 minutos. Actividad 6. Evaluación final. Cuestionario de redacción abierta, con preguntas similares al cuestionario de ideas iniciales.

Tiempo estimado: 30 minutos.
Sintesis y conclu- $\quad$ - Intervención en siones del tema estudiado en este ciclo de mejora.

\section{Cuestionario inicial - final}

Se ha realizado un cuestionario inicial de 5 preguntas con 4 opciones para elegir una de ellas, pudiendo razonar de forma escrita la respuesta elegida, de manera que el alumno pudiera guiarse desde sus propios conocimientos. Hay preguntas acerca de sus creencias, de sus prejuicios ante los tópicos de los trastornos del desarrollo en la sociedad, así como de sus conocimientos más profundos sobre contenidos concretos.

Al finalizar las actividades, se administra un cuestionario de 5 preguntas también, en este caso con respuestas de tipo abiertas, con los mismos contenidos e ideas que en el cuestionario inicial, para determinar el grado de evolución de los aprendizajes de los estudiantes. 


\section{Aplicación del Ciclo de Mejora Docente}

\section{Relato de las sesiones}

Actividad 1. Exploración de ideas. Esta actividad se llevó a cabo sin dificultades y en el tiempo estimado inicialmente.

Actividad 2. Teoría/Debate. Las ideas iniciales respecto a algunos de los trastornos del desarrollo (síndrome de Down), exploradas en la actividad anterior, estaban llenas de prejuicios y tópicos por parte del alumnado, que además desconocía el desarrollo específico en los primeros años de vida. Al igual ocurrió con el TEA, conocían la sintomatología general en base a los tópicos específicos de estos trastornos. Se creó un debate muy enriquecedor y surgieron muchas preguntas por parte del alumnado en esta actividad. El tiempo estimado también se cumplió porque finalizaba el tiempo de dicha clase y tenían clase después de otra asignatura; pero el debate quedó bien zanjado.

Actividad 3. Actividad de planificación y diseño de intervención. Esta actividad ha sido muy enriquecedora para los alumnos. Ha sido un trabajo realizado en clase, en base a los apuntes teóricos de la misma (disponibles previamente en la plataforma de enseñanza virtual), pero sobre los que han tenido que trabajar intensamente durante dos horas en sesión de aula. Han contado con mi apoyo como profesora durante todo el tiempo para orientarles en el trabajo. Las dos horas de trabajo en clase han sido suficientes para la elaboración de dicho trabajo. Solamente un grupo ha precisado terminar la tarea en casa. Las exposiciones de los pequeños grupos me han sorprendido muy gratamente, tanto por el contenido como por la forma de las mismas, así como por la seguridad y el ambiente generado en el aula durante las exposiciones. Necesitamos tomar 15 minutos más de la hora estimada en esta actividad. 
Actividad 4. Análisis de caso. Esta actividad ha sido levemente modificada. En lugar de analizar un caso por escrito y detectar los signos de alarma, la hemos modificado hacia la siguiente actividad: hemos visto el vídeo del caso del niño con TEA y los alumnos, en pequeños grupos de unas 4 o 5 personas, han tenido que detectar las señales de alarma en todas las áreas del desarrollo y plasmarlas en cartulinas, cada grupo de un área específica, de manera que, al finalizar esta tarea, se han puesto en común y se ha lanzado la pregunta final: ¿qué puede hacer el fisioterapeuta en este trastorno? Se ha creado un debate muy enriquecedor y una síntesis importante del tema teórico. El tiempo estimado se ha visto aumentado en 20 minutos (1 hora y 20 minutos).

Actividad 5. Elaboración de conclusiones. Los alumnos han mostrado un gran interés por este tema. No he tenido faltas de asistencia en las clases teóricas en estas casi tres semanas del ciclo de mejora y los alumnos se han implicado en todas las actividades. Han sabido responder a la pregunta lanzada para establecer las conclusiones de una manera muy correcta y detallada, en casi todos los casos. Estoy muy orgullosa de la evolución de las clases, así como de la implicación y participación del alumnado. Hemos cumplido el tiempo marcado para dicha actividad.

Actividad 6. Evaluación final. Hemos administrado el cuestionario final y lo han cumplimentado todos los alumnos excepto una alumna que no pudo hacerlo, por enfermedad. El tiempo estimado se ha visto disminuido, ya que solo hemos precisado 15 minutos para esta actividad.

\section{Evaluación del aprendizaje de los estudiantes}

Los criterios que se han utilizado para evaluar al alumnado han sido los siguientes:

- Asocia contenidos específicos de este tema (trastornos del desarrollo) a los aprendidos en temas anteriores (intervención en las distintas áreas del desarrollo, evaluación y trabajo general de Atención Temprana). 
- Conoce las características específicas del desarrollo, objetivos y herramientas de intervención en síndrome de Down, prematuridad, atrofia muscular espinal, distrofia muscular de Duchenne y acondroplasia.

- Argumenta y razona la organización y planificación de un programa de intervención en Atención Temprana.

- Expresa con claridad la fundamentación de dicha propuesta de intervención.

Estos criterios se han mantenidos en todos los instrumentos de evaluación. A continuación, desarrollamos los resultados obtenidos, más concretamente, a partir del análisis de los cuestionarios iniciales y finales de los alumnos, según la escalera de progresión que hemos establecido para el análisis e interpretación de dichos resultados.
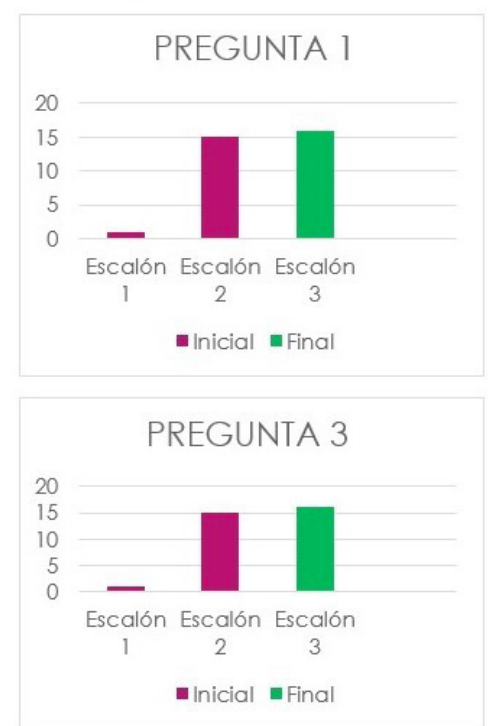
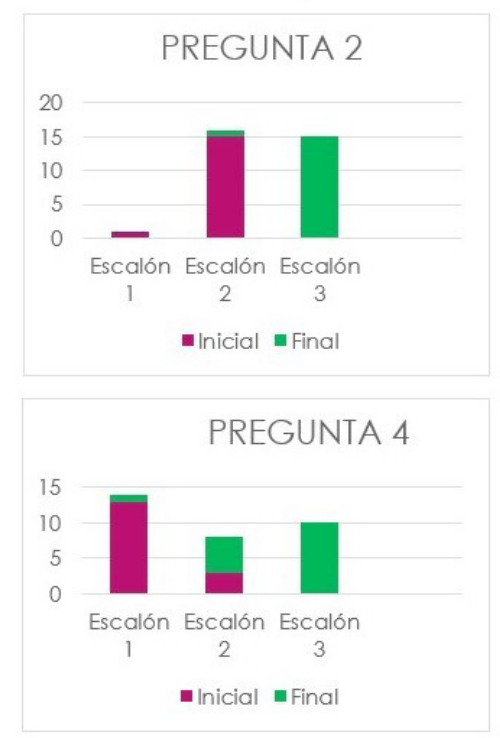

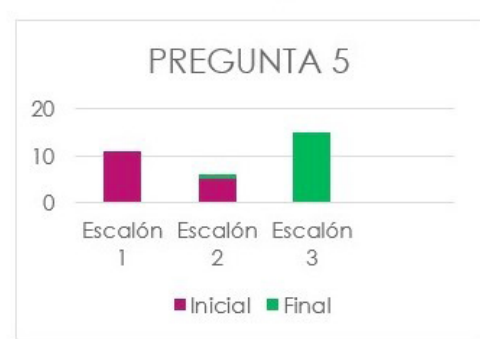

Figura 3. Resultados de la evaluación de los aprendizajes de los estudiantes en las 5 preguntas de los cuestionarios inicial y final.

Jornadas de Formación e Innovación Docente del Profesorado | № 1(2018) 
En la Figura 3 exponemos los resultados obtenidos, expresados en números absolutos y reflejados en gráficos, en las respuestas de los alumnos a los cuestionarios iniciales y finales en cada uno de los escalones de la escalera. El total de alumnos que han participado en el cuestionario inicial y final ha sido de 16 alumnos de los 18 matriculados en la asignatura. Los dos alumnos que faltan no asistieron al cuestionario inicial o al final en alguno de los casos. Hemos dividido cada pregunta en tres escalones graduales de posibles respuestas: escalón 1: no responde correctamente lo que se le pregunta (no existe idea o lo que se dice no tiene sentido); escalón 2: responde correctamente pero no argumenta su respuesta (idea, pero sin justificación); y escalón 3: responde correctamente y argumenta su respuesta.

Se desprende de los resultados sobre la evolución o progresión de los aprendizajes de los alumnos que todos han subido de escalón en todas las preguntas. En muchos casos incluso algún alumno ha avanzado del escalón número 1 al escalón número 3, por lo que podemos considerar la evolución de los aprendizajes generales de los alumnos como positiva.

\section{Evaluación del Ciclo de Mejora Docente}

\section{Evaluación del modelo metodológico}

Se administró una encuesta sobre la metodología de enseñanza llevada a cabo, de manera que los alumnos pudieran evaluar las habilidades de la profesora y las técnicas o herramientas utilizadas para el desarrollo de este tema.

Tras la administración de la evaluación del ciclo de mejora y de la docente a los 14 alumnos presentes en el aula, participantes todos en el ciclo de mejora, los resultados obtenidos son los que siguen. 
1. ¿Cómo evaluarías, del 1 al 10, la metodología empleada en la asignatura de Atención Temprana?

El 5\% respondió un 8 , el $80 \%$ un 9 y el $15 \%$ un 10.

2. ¿Cómo evaluarías, del 1 al 10, el papel de la profesora para dinamizar y organizar sus clases?

El 15\% contestó un 9, y el 85\% un 10.

3. ¿Qué actividades te han gustado más y por qué? (Vídeos y comentarlos, resúmenes en cartulinas, preparación en clase y exposición de casos concretos, pistas y resolución en grupos...): visualización de vídeos y debates: 92.9\%; resúmenes en cartulinas: $42.9 \%$; preparación de trastorno y exposición grupal: 35.7\%; resolución de problemas a través de pistas (taller conceptual): $28.6 \%$; y no especifica: $21.4 \%$.

4. Valora, del 1 al 10, cuál crees que ha sido tu aprendizaje de la materia, atendiendo a esta metodología de enseñanza: el 30\% lo valora como un 8 , el $50 \%$ como un 9 y el $20 \%$ como un 10.

5. Comentarios/sugerencias/críticas: Los comentarios, en general, han sido como una valoración general del ciclo de mejora y de la asignatura en general. Algunas ideas importantes que se han repetido son que la metodología docente ha sido favorecedora del aprendizaje y que la participación constante del alumnado ameniza la docencia y ayuda a la implicación y reflexión de la asignatura.

\section{Cuestiones a mantener y cambios a introducir}

Las actividades realizadas no han sido novedosas en mi práctica docente, ya que las venía realizando, aunque no de manera tan organizada y con un sentido tan claro. Pero mi organización respecto a las mismas, el sentido que les he dado y el concepto que tenía acerca de algunas metodologías concretas sí que han cambiado. 
La evaluación inicial la considero ahora muy necesaria para conocer los esquemas mentales del alumnado y permite a la profesora adaptarse a sus ideas iniciales. La evaluación como medida del progreso y no como el fin a donde llega cada alumno, contemplando la propia diversidad de aprendizaje que existe en nuestro alumnado, es un factor también a mantener a partir de esta experiencia.

He aprendido a "dar clase con la boca cerrada" (Finkel, 2008) y a generar un ambiente para el "aprendizaje crítico natural" (Bain, 2004), dando paso totalmente a la participación del alumnado, haciéndoles responsables de su propio aprendizaje. También he aprendido a ser más flexible en el desarrollo de la teoría y de la práctica, entendiendo las diversas relaciones que puede haber entre estas dos dimensiones del conocimiento. Ahora tengo en cuenta todas las opciones docentes según las necesidades del grupo y que puedan tener los alumnos un aprendizaje más reflexivo y participativo.

\section{Aspectos que se pretenden incorporar a la práctica docente habitual}

En nuestra práctica docente pretendemos continuar con el diseño metodológico planteado, centrado en una filosofía de trabajo participativa, donde la reflexión y las ganas de mejorar sean piezas clave de nuestro propio proceso de aprendizaje. También mantendré la organización a través de los mapas de contenidos, problema y actividades, pues me permite ser más flexible y adaptable a las necesidades de aprendizaje de los alumnos.

Otro aspecto a considerar en mi futura práctica docente son los llamados "talleres conceptuales" (Finkel, 2008), utilizados de forma más frecuente y no como una actividad aislada, puesto que el nivel de respuesta y participación del alumnado ha sido excelente; es una actividad motivadora a la par que creativa y que propicia un importante nivel de implicación y reflexión del alumnado. 


\section{Principios didácticos argumentados}

La realización del Curso General de Docencia Universitaria y la experiencia con los ciclos de mejora me han llevado a plantearme una serie de principios en mi docencia:

- Es importante resaltar los contenidos conceptuales, procedimentales y actitudinales como contenidos con la misma relevancia para el desarrollo del aprendizaje completo con vistas al desempeño de la profesión de fisioterapeuta en Atención Temprana.

- La participación del alumnado, a través de la reflexión y la crítica, debe formar parte del día a día de mis clases.

- Es imprescindible conocer los esquemas mentales del alumnado al inicio, así como la progresión de su aprendizaje individualizado, y no tanto una calificación numérica.

- Las clases serán un punto de encuentro entre el docente y el estudiante para compartir aprendizajes, resolver problemas, debatir experiencias y facilitar reflexión.

\section{Referencias bibliográficas}

Bain, K. (2004). Lo que hacen los mejores profesores universitarios. Valencia: Publicaciones de la Universidad de Valencia.

De Alba, N. y Porlán, R. (2017). La metodología de enseñanza. En R. Porlán (coord.), Enseñanza universitaria. Cómo mejorarla (pp. 37-54). Madrid: Morata.

Finkel, D. (2008). Dar clase con la boca cerrada. Valencia: Publicaciones de la Universidad de Valencia.

Jornadas de Formación e Innovación Docente del Profesorado | № 1 (2018) Esta obra se distribuye con la licencia Creative Commons 